\title{
THE AREA AND VARIATION OF LINEARLY CONTINUOUS FUNCTIONS
}

\author{
WILLIAM P. ZIEMER ${ }^{1}$
}

1. Introduction. Casper Goffman has developed a theory of area for functions of several variables whose gradients are totally finite vectorvalued measures, [G1], [G2], [G3]. In the special case of linearly continuous functions of two variables, he has shown in [G2] that the area is equal to the Hausdorff 2-dimensional measure of the "essential" part of the graph. A similar result is valid for linearly continuous functions of several variables (3.6). In this paper, a co-area formula is established (3.7) which relates the integral of the Hausdorff $n-1$ dimensional measure of the slices by hyperplanes of the essential portion of the graph to the total variation of the gradient measure. In other situations, this has been shown to be a very useful tool, [F3], [GE'], [Z1], [Z2].

Our results have also been established independently by Herbert Federer by different methods. His results will be incorporated in his forthcoming book Geometric measure theory.

2. Notation and preliminaries. Throughout, $x=\left(x_{1}, \cdots, x_{n}\right)$ will be an arbitrary point of Euclidean $n$-space $E_{n} . L_{n}$ will denote $n$-dimensional Lebesgue measure and for $k \leqq n, H^{k}$ and $I^{k}$ will stand for $k$ dimensional Hausdorff measure and $k$-dimensional integralgeometric measure respectively. In particular, $H^{0}(A)$ is the number of points in the set $A$. Recall that $H^{k}$ and $I^{k}$ agree on the $\left(H^{k}, k\right)$ rectifiable sets of finite $H^{k}$ measure, cf. [F2, Theorem 9.7]. $G_{n}$ is the orthogonal group on $E_{n}$ and $\phi_{n}$ the Haar measure on $G_{n}$ such that $\phi_{n}\left(G_{n}\right)=1$. With $R \in G_{n}$ and $w \in E_{n-1}$ associate the line $\lambda(R, w)$ given by

$$
\lambda(R, w)=R\left(E_{n} \cap\left\{x: x_{i}=w_{i}, i=1,2, \cdots, n-1\right\}\right) .
$$

Under the map $\lambda$, the image of the product measure $\phi_{n} \times L_{n-1}$ is the Haar measure on the space of all lines in $E_{n}$.

Let BV stand for the space of all locally summable functions on $E_{n}$ whose every distribution theory partial derivative is a totally finite measure. For $f \in \mathrm{BV}$, these measures will be denoted by $D_{1}(f), \cdots, D_{n}(f)$. A function is called linearly continuous if, in some

Presented to the Society, May 18, 1967 under the title The area and variation of linearly continuous functions on $E^{n}$; received by the editors October $6,1967$.

1 This work was supported in part by a grant from the National Science Foundation. 
coordinate system, $f$ is continuous on almost all lines parallel to the coordinate axes. Functions of compact support which are BV and linearly continuous will be the objects of interest in this paper. Denote this class of functions by $\mathscr{L}$. In [G3], Goffman established the remarkable fact that functions in $\mathscr{L}$ are invariant under bilipschitzian maps. In particular, a function $f \in \mathscr{L}$ is linearly continuous with respect to all rectangular coordinate systems. Thus, we have the following

2.1. Theorem. If $f \in \mathscr{L}$ and $R \in G_{n}$, then $f$ is continuous on $\lambda(R, w)$ for $L_{n-1}$ almost every $w \in E_{n-1}$.

For $f \in \mathscr{L}$, consider the set of integral means

$$
f_{k}(x)=L_{n}\left[S\left(x, k^{-1}\right)\right]^{-1} \int_{S(x, k-1)} f(y) d L_{n}(y),
$$

where $S\left(x, k^{-1}\right)$ is the $n$-ball of radius $k^{-1}$ and center $x$. Let $\beta_{f}$ be the total variation of the gradient measure $\left(D_{1}(f), \cdots, D_{n}(f)\right)$ and $\alpha_{f}$ the total variation of $\left(L_{n}, D_{1}(f), \cdots, D_{n}(f)\right)$. If $\beta_{k}$ and $\alpha_{k}$ are the corresponding measures for $f_{k}$, then for every open rectangle $S \subset E_{n}$, it was shown in $[\mathrm{G} 3$, Lemma 1] that

$$
\lim _{k \rightarrow \infty} \alpha_{k}(S)=\alpha_{f}(S), \quad \lim _{k \rightarrow \infty} \beta_{k}(S)=\beta_{f}(S) .
$$

Also, for $k=1,2, \cdots$, and $i=1,2, \cdots, n$,

$$
\beta_{k}\left(S_{k}\right) \leqq \beta_{f}(S) \text { and }\left|D_{i}\left(f_{k}\right)\right|\left(S_{k}\right) \leqq\left|D_{i}(f)\right|(S),
$$

where $\left|D_{i}(f)\right|$ denotes the total variation of the measure $D_{i}(f)$, and where $S_{k}$ is the set of points in $S$ whose distance from $E_{n}-S$ is greater than $k^{-1}$.

3. Co-area and integralgeometric measure. Throughout the remainder of the paper, we will consider a function $f \in \mathscr{L}$ defined on the open unit cube $Q$. Since the integral means $f_{k}$ are BV and continuous, they are of bounded variation in the sense of Tonelli (BVT) and therefore, the proof of Theorem 3.1 in [ $\mathrm{Z1}$ ] gives

(4) $\beta_{k}(Q)=\kappa \int_{G_{n}} \int_{E_{n-1}} \int_{E_{1}} H^{0}\left[f_{k}^{-1}(t) \cap \lambda(R, w)\right] d L_{1}(t) d L_{n-1}(w) d \phi_{n}(R)$,

where $\kappa$ is the normalizing constant used in the definition of $n-1$ dimensional integralgeometric measure $[F 2,2.16]$. In view of the Banach indicatrix formula for the variation of continuous functions of a real variable, it follows from [G3, p. 169] that 


$$
\left|D_{n}(f)\right|(Q)=\int_{E_{n-1}} \int_{E_{1}} H^{0}\left[f^{-1}(t) \cap \lambda\left(R_{0}, w\right)\right] d L_{1}(t) d L_{n-1}(w),
$$

where $R_{0}: E_{n} \rightarrow E_{n}$ is the identity. Thus, from (3) and the coordinate invariance of $f$, for every $R \in G_{n}$,

$$
\begin{aligned}
\left|D_{n}\left(f_{k} \circ R\right)\right|\left(Q_{k}\right) & \leqq\left|D_{n}(f \circ R)\right|(Q) \\
& =\int_{E_{n-1}} \int_{E_{1}} H^{0}\left[f^{-1}(t) \cap \lambda(R, w)\right] d L_{1}(t) d L_{n-1}(w) \\
& \leqq \beta_{f}(Q) .
\end{aligned}
$$

But $\left|D_{n}\left(f_{k} \circ R\right)\right|\left(Q_{k}\right) \rightarrow\left|D_{n}(f \circ R)\right|(Q)$ for every $R \in G_{n}$, and thus, Lebesgue's dominated convergence theorem along with (2), (4), and (5) leads to

(6) $\beta_{f}(Q)=\kappa \int_{G_{n}} \int_{E_{n-1}} \int_{E_{1}} H^{0}\left[f^{-1}(t) \cap \lambda(R, w)\right] d L_{1}(t) d L_{n-1}(w) d \phi_{n}(R)$.

A similar result would have been obtained if $f$ had been restricted to an open rectangle of $Q$. Every open set is the union of nonoverlapping closed rectangles and $\beta_{f}$ vanishes on the boundary of every rectangle, [G3, \$7]. Also, whether open or closed rectangles are used will not affect the value of the integral in (6). Thus, we have

3.1. Lemma. If $f \in \mathcal{L}$ is defined on $Q$ and $U$ is an open subset of $Q$, then

$\beta_{f}(U)=\kappa \int_{G_{n}} \int_{E_{n-1}} \int_{E_{1}} H^{0}\left[f^{-1}(t) \cap U \cap \lambda(R, w)\right] d L_{1}(t) d L_{n-1}(w) d \phi_{n}(R)$.

If $B \subset Q$ is a Borel set for which $\beta_{f}(B)=0$, then for every $\epsilon>0$ there is an open set $U \supset B$ such that $\beta_{f}(U)<\epsilon$. Thus, the preceding lemma leads directly to

3.2. LEMмA. If $B$ is a Borel set for which $\beta_{f}(B)=0$, then

$$
\kappa \int_{G_{n}} \int_{E_{n-1}} \int_{E_{1}} H^{0}\left[f^{-1}(t) \cap B \cap \lambda(R, w)\right] d L_{1}(t) d L_{n-1}(w) d \phi_{n}(R)=0 .
$$

For a set $A \subset Q$, let $A^{i}$ denote the projection of $A$ into $E_{n-1}$ in the $x_{i}$ direction. The following fact was very useful in [G3]: for every positive integer $k$ there is a closed set $T_{k}^{i} \subset Q^{i}$ with the property that, if $T_{k}=\bigcup_{i=1}^{n} T_{k}^{i} \times[0,1]$, then 
(i) $f$ is continuous on $T_{k}$,

(ii) $L_{n-1}\left(Q^{i}-T_{k}^{i}\right)<k^{-1}, \quad i=1,2, \cdots, n$,

(iii) $\alpha_{f}\left(Q-T_{k}\right)<k^{-1}$.

Let

$$
E_{f}=U T_{k} \text { and } Z_{f}=Q-E_{f} .
$$

Notice that $\alpha_{f}\left(Z_{f}\right)=\beta_{f}\left(Z_{f}\right)=L_{n}\left(Z_{f}\right)=0$ and that $Z_{f}$ projects into a set of $L_{n-1}$ measure zero in each coordinate direction. It will be shown that $f$ restricted to $E_{f}$ describes the essential behavior of $f$ on $Q$. As a first step in this direction, we see that 3.1 and 3.2 imply

$\beta_{f}(Q)$

$$
=\kappa \int_{G_{n}} \int_{E_{n-1}} \int_{E_{1}} H^{0}\left[f^{-1}(t) \cap E_{f} \cap \lambda(R, w)\right] d L_{1}(t) d L_{n-1}(w) d \phi_{n}(R) .
$$

3.3. Definition. With the function $f \in \mathscr{L}$, associate the function $f^{*}: Q \rightarrow E_{n+1}$ defined by $f\left(x_{1}, \cdots, x_{n}\right)=\left(x_{1}, \cdots, x_{n}, f\left(x_{1}, \cdots, x_{n}\right)\right)$. Also, for every set $A \subset Q$, let $A^{*}=f^{*}(A)$.

It is clear that $E_{f}^{*}=f^{*}\left(E_{f}\right)$ is an analytic (in fact, Borel) subset of $E_{n+1}$. Thus, for every $t \in E_{1}, f^{-1}(t) \cap E_{f}$ is an analytic set of $E_{n}$, since it is the projection of the intersection of $E_{f}^{*}$ with a hyperplane at height $t$. Since $f^{-1}(t) \cap E_{f}$ is an analytic set, [F2, 5.11] gives

$$
\begin{aligned}
I^{n-1}\left[f^{-1}(t)\right. & \left.\cap E_{f}\right] \\
= & \kappa \int_{G_{n}} \int_{E_{n-1}} H^{0}\left[f^{-1}(t) \cap E_{f} \cap \lambda(R, w)\right] d L_{n-1}(w) d \phi_{n}(R) .
\end{aligned}
$$

Assuming for the moment that the integrand in (9) is $L_{1} \times L_{n-1} \times \phi_{n}$ measurable, (9), (10), and Fubini's theorem yield the following expression for $\beta_{f}$ :

$$
\beta_{f}(Q)=\int_{E_{1}} I^{n-1}\left[f^{-1}(t) \cap E_{f}\right] d L_{1}(t) .
$$

To see that the integrand in (9) is $L_{1} \times L_{n-1} \times \phi_{n}$ measurable, consider the projection $P: E_{n+1} \rightarrow E_{n}$ defined by

$$
P\left(x_{1}, \cdots, x_{n-1}, x_{n}, x_{n+1}\right)=\left(x_{1}, \cdots, x_{n-1}, x_{n+1}\right) .
$$

For $R \in G_{n}$, let $R^{\prime}: E_{n+1} \rightarrow E_{n+1}$ be defined by $R^{\prime}\left(x_{1}, \cdots, x_{n+1}\right)$ $=\left(x_{1}^{\prime}, \cdots, x_{n}{ }^{\prime}, x_{n+1}\right)$, where $R\left(x_{1}, \cdots, x_{n}\right)=\left(x_{1}^{\prime}, \cdots, x_{n}^{\prime}\right)$. Since $E_{f}^{*}$ is an analytic set, there is a standard procedure to show that the multiplicity function $N\left[P R^{\prime} f^{*}, E_{f}, y\right]$ is $L_{n} \times G_{n}$ measurable, cf. 
$[\mathbf{F 2}, 2.17]$. Here, $N\left[P R^{\prime} f^{*}, E_{f}, y\right]$ means the number of points in $E_{f}$ that are mapped into $y$ by $P R^{\prime} f^{*}$. If $y$ is written as $(w, t)$ then $N\left[P R^{\prime} f^{*}, E_{f},(w, t)\right]$ is $L_{1} \times L_{n-1} \times \phi_{n}$ measurable. But

$$
N\left[P R^{\prime} f^{*}, E_{f},(w, t)\right]=H^{0}\left[f^{-1}(t) \cap E_{f} \cap \lambda(R, w)\right]
$$

and thus, (11) is established.

Define a Borel measure $\nu_{f}$ by

$$
\nu_{f}(B)=\int_{E_{1}} I^{n-1}\left[f^{-1}(t) \cap B \cap E_{f}\right] d L_{1}(t),
$$

the $L_{1}$ measurability of the integrand being established as above. Then, by the same reasoning used to obtain (11), it is clear that $\beta_{f}$ and $\nu_{f}$ agree on all open rectangles and therefore, on all Borel sets. Therefore, we have proved the following theorem.

3.4. TheOREM. If $f \in \mathfrak{L}$ and $E_{f}$ is the set defined in (8), then

$$
\beta_{f}(B)=\int_{E_{1}} I^{n-1}\left[f^{-1}(t) \cap B \cap E_{f}\right] d L_{1}(t)
$$

for every Borel set $B$.

It follows from Stepanoff's Theorem that a function $f \in \mathfrak{L}$ has an approximate total differential $L_{n}$ almost everywhere and thus, by [F1], there are disjoint Borel sets $A_{0}, A_{1}, \cdots$ whose union is $Q$ with $L_{n}\left(A_{0}\right)=0$ such that $f$ is Lipschitz on $A_{k}, k=1,2, \cdots$. Therefore, the following theorem has some interest.

3.5. Theorem. If $f \in \mathcal{L}$ is Lipschitz on a Borel set $B \subset Q$, then $H^{n}\left(B^{*}\right)=\alpha_{f}(B)$.

Proof. For each open rectangle $S \subset Q$, it follows from Proposition 7 in $[\mathrm{G} 1]$ that

$$
\alpha_{f}(S) \geqq \int_{S}\left(1+\left|\nabla f_{a p}\right|^{2}\right)^{1 / 2} d L_{n} .
$$

Thus, the same inequality must be valid for all Borel sets. By considering a Lipschitz extension of $f$ on $B$ to all of $E_{n}$ [RR, p. 341], we have from $[F 2,5.9]$ that

$$
\int_{B}\left(1+\left|\nabla f_{a p}\right|^{2}\right)^{1 / 2} d L_{n}=H^{n}\left[f^{*}(B)\right]=H^{n}\left(B^{*}\right) .
$$

Thus, 


$$
\alpha_{f}(B) \geqq H^{n}\left(B^{*}\right) .
$$

To obtain the opposite inequality, let $F$ be the Lipschitz extension of $f$ on $B$ to all of $E_{n}$ and recall from $[\mathrm{F} 3,3.1]$ that

$$
\begin{aligned}
\int_{B \cap E_{f}}\left|\nabla f_{a p}\right| d L_{n} \\
\quad=\int_{B \cap E_{f}}|\nabla F| d L_{n}=\int_{E_{1}} H^{n-1}\left[F^{-1}(t) \cap B \cap E_{f}\right] d L_{1}(t) \\
=\int_{E_{1}} H^{n-1}\left[f^{-1}(t) \cap B \cap E_{f}\right] d L_{1}(t) .
\end{aligned}
$$

Since $\left(B \cap E_{f}\right)^{*}=f^{*}\left(B \cap E_{f}\right)$ if $\left(H^{n}, n\right)$ is rectifiable, by appealing again to the proof of 3.1 in $\left[Z_{1}\right]$, it follows that $f^{-1}(t) \cap B \cap E_{f}$ is $\left(H^{n-1}, n-1\right)$ rectifiable for $L_{1}$ almost for all $t \in E_{1}$. Thus, (3.4) and (14) yield for every Borel set $B$,

$$
\begin{aligned}
\beta_{f}\left(B \cap E_{f}\right) & =\int_{E_{1}} H^{n-1}\left[f^{-1}(t) \cap B \cap E_{f}\right] d L_{1}(t) \\
& =\int_{B \cap_{E_{f}}}\left|\nabla f_{a p}\right| d L_{n} .
\end{aligned}
$$

As in [G1, §6], for every $\epsilon>0$, there is a finite Borel partition $\left\{B_{k}\right\}$ of $B \cap E_{f}$ such that

$$
\sum_{k}\left[L_{n}\left(B_{k}\right)^{2}+\left(\int_{B_{k}}\left|\nabla f_{a p}\right|\right)^{2}\right]^{1 / 2}<\int_{B \cap_{E_{f}}}\left(1+\left|\nabla f_{a p}\right|^{2}\right)^{1 / 2}+\epsilon / 2
$$

and

$$
\alpha_{f}\left(B \cap E_{f}\right)<\sum_{k}\left(L_{n}\left(B_{k}\right)^{2}+\beta_{f}\left(B_{k}\right)^{2}\right)^{1 / 2}+\epsilon / 2 .
$$

Hence, from (15) and $[\mathbf{F 2}, 5.9]$,

$$
\alpha_{f}\left(B \cap E_{f}\right)<H^{n}\left[\left(B \cap E_{f}\right)^{*}\right]+\epsilon .
$$

Since $\epsilon$ is arbitrary, (13) and (16) imply

$$
B^{n}\left[\left(B \cap E_{f}\right)^{*}\right] \leqq H^{n}\left(B^{*}\right) \leqq \alpha_{f}(B)=\alpha_{f}\left(B \cap E_{f}\right) \leqq H^{n}\left[\left(B \cap E_{f}\right)^{*}\right],
$$

which establishes the lemma.

Let $G=Q \times E_{1} \cap\{(p, t): t \leqq f(p)\}$ and let $P(G)$ denote those points at which the exterior normal to $G$ exists [F4]. Define the map $\pi: E_{n+1} \rightarrow E_{n}$ by $\pi\left(x_{1}, \cdots, x_{n+1}\right)=\left(x_{1}, \cdots, x_{n}\right)$. The following result 
was obtained independently by the author and Herbert Federer. Since our proof is somewhat similar to Federer's, it will not be given here. Federer's proof will appear in his forthcoming book Geometric measure theory.

3.6. Theorem. If $f$ is $B V$, then

$$
\alpha_{f}(B)=H^{n}\left[P(G) \cap \pi^{-1}(B)\right]
$$

for every Borel set $B \subset Q$.

In the case that $f \in \mathcal{L}$, proceeding as in $[\mathbf{G} 3, \S 6]$, it is easy to find a Borel set $T \subset Q$ such that $\alpha_{f}(Q-T)=0$ and $f$ is approximately continuous at every point of $T$. Thus, letting $A_{f}=T \cap \pi[P(G)] \cap E_{f}$, we have $\alpha_{f}\left(Q-A_{f}\right)=0$ and it is simple to verify that $A_{f}^{*} \subset P(G) . P(G)$ is $\left(H^{n}, n\right)$ rectifiable and thus so is $A_{f}^{*}$. It follows that $f^{-1}(t) \cap A_{f}$ is $\left(H^{n-1}, n-1\right)$ rectifiable for $L_{1}$ almost all $t \in E_{1}$, and therefore the following theorem follows directly from 3.4 .

3.7. Theorem. If $f \in \mathcal{L}$, then

$$
\beta_{f}(B)=\int H^{n-1}\left[f^{-1}(t) \cap B \cap A_{f}\right] d L_{1}(t)
$$

for every Borel set $B \subset Q$.

\section{BIBLIOGRAPHY}

[F1.] H. Federer, Surface area. II, Trans. Amer. Math. Soc. 55 (1944), 438-456.

[F2.] - The $(\phi, K)$ rectifiable subsets of $n$ space, Trans. Amer. Math. Soc. 62 (1947), 114-192.

[F3.] - Curvature measures, Trans. Amer. Math. Soc. 93 (1959), 418-491.

[F4.] —, A note on the Gauss-Green theorem, Proc. Amer. Math. Soc. 9 (1958), $447-451$.

[GE.] F. Gehring, Extremal length definitions for the conformal capacity in space, Michigan Math. J. 9 (1962), 137-150.

[G1.] C. Goffman, Lower-semi-continuity and area functionals, Rend. Circ. Mat. Palermo (2) 2 (1954), 203-235.

[G2.] - Nonparametric surfaces given by linearly continuous functions, Acta Math. 103 (1960), 269-291.

[G3.] - A characterization of linearly continuous functions whose partial derivatives are measures, Acta Math. 117 (1967), 165-190.

[RR.] T. Rado and P. V. Reichelderfer, Continuous transformations in analysis, Grundlehren der mathematischen Wissenschaften, Springer, Berlin, 1955.

[Z1.] W. Ziemer, Some lower bounds for Lebesgue area, Pacific J. Math. (2) 19 (1966).

[Z2.] - Extremal length and conformal capacity, Trans. Amer. Math. Soc. 126 (1967), 460-473.

INDIANA UNIVERSITY 